\title{
THE DUALITY OPERATION IN THE CHARACTER RING OF A FINITE CHEVALLEY GROUP
}

\author{
BY DEAN ALVIS
}

It is possible (as in [4]) to define a duality operation $\zeta \rightarrow \zeta^{*}$ in the ring of virtual characters of an arbitrary finite group with a split $(B, N)$-pair of characteristic $p$. Such a group arises as the fixed points under a Frobenius map of a connected reductive algebraic group, defined over a finite field [1]. This paper contains statements of several general properties of the duality map $\zeta \rightarrow \zeta^{*}$ and two related operations (see $\S \S 2$ and 4 ). The duality map $\zeta \rightarrow \zeta^{*}$ generalizes the construction in [2] of the Steinberg character, and interacts well with the organization of the characters from the point of view of cuspidal characters (§6). It is hoped that there is also a useful interaction with the Deligne-Lusztig virtual characters $R_{\mathrm{T}}^{\mathrm{G}} \theta$. Partial results have been obtained in this direction ( $\$ 5$ ). Detailed proofs will appear elsewhere.

1. Let $G$ be a finite group with split $(B, N)$-pair of characteristic $p$. Let $(W, R)$ be the Coxeter system, and let $P_{J}=L_{J} V_{J}$ be the standard parabolic subgroup corresponding to $J \subseteq R$, with $V_{J}=O_{P}\left(P_{J}\right)$ (see [3] for definitions and notations). Let $\operatorname{char}(G)$ denote the ring of virtual characters of $G$, and $\operatorname{Irr}(G)$ the set of irreducible characters of $G$, all taken in the complex field. For $J \subseteq R$ and $\zeta \in \operatorname{char}(G)$ define

$$
\zeta_{\left(P_{J} / V_{J}\right)}=\Sigma\left(\zeta, \tilde{\lambda}^{G}\right)_{\mathrm{G}} \lambda
$$

where $\sim$ denotes extension to $P_{J}$ via the projection $P_{J} \rightarrow L_{J} \cong P_{J} / V_{J}$, and the sum is over all $\lambda \in \operatorname{Irr}\left(L_{J}\right)$. Let $\zeta_{\left(P_{J}\right)}=\zeta_{\left(P_{J} / V_{J}\right)} \sim$. The duality map is then defined by:

1.2 Definition. $\zeta^{*}=\Sigma_{J \subseteq R}(-1)^{|J|} \zeta_{\left(P_{J}\right)}{ }^{G}$, for all $\zeta \in \operatorname{char}(G)$.

2. The truncation map $\zeta \rightarrow \zeta_{\left(P_{J} / V_{J}\right)}$ and the map $\lambda \rightarrow \tilde{\lambda}^{G}$ behave in much the same way as ordinary restriction and induction. The following basic properties follow directly from the structure theorems [3].

2.1 Frobenius ReCIPRocity. Let $\zeta \in \operatorname{char}(G)$ and $\lambda \in \operatorname{char}\left(L_{J}\right)$. Then

Received by the editors May 7, 1979.

AMS (MOS) subject classification numbers (1970). Primary $20 \mathrm{C1} 5$. 


$$
\left(\zeta, \tilde{\lambda}^{G}\right)_{G}=\left(\zeta_{\left(P_{J}\right)}, \widetilde{\lambda}\right)_{P_{J}}=\left(\zeta_{\left.P_{J} / V_{J}\right)}, \lambda\right)_{L_{J}} .
$$

2.2 Transitivity. If $K \subseteq J \subseteq R$, let $Q_{K}$ be the standard parabolic subgroup $P_{K} \cap L_{J}$ of $L_{J}$ and let $V_{J, K}=O_{p}\left(Q_{K}\right)=L_{J} \cap V_{K}$. Then if $\zeta \in$ $\operatorname{char}(G)$ and $\zeta \in \operatorname{char}\left(L_{J}\right)$, we have

and

$$
\left(\zeta_{\left(P_{J} / V_{J}\right)}\right)\left(Q_{K} / V_{J, K}\right)=\zeta_{\left(P_{K} / V_{K}\right)},
$$

$$
\left(\tilde{\lambda}^{L}\right)^{\sim G}=\tilde{\lambda}^{G}
$$
Then

2.3 INTERTWINING NUMBER THEOREM. Let $\lambda_{i} \in \operatorname{char}\left(L_{J_{i}}\right)$ for $i=1,2$.

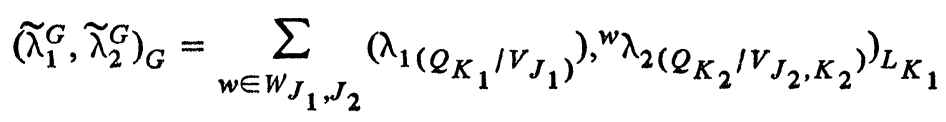

where $W_{J_{1}, J_{2}}$ is the set of distinguished $W_{J_{1}}-W_{J_{2}}$ double coset representatives, $W_{K_{1}}=W_{J_{1}} \cap{ }^{w} W_{J_{2}}$ and $W_{K_{2}}=W_{J_{2}} \cap{ }^{w^{1}-1} W_{J_{1}}$.

2.4 Subgroup Theorem. Let $\lambda \in \operatorname{char}\left(L_{J_{1}}\right)$. Then

$$
\left(\tilde{\lambda}^{G}\right)_{\left(P_{J_{2}} / V_{J_{2}}\right)}=\sum_{w \in W_{J_{1}, J_{2}}} w^{-1}\left(\lambda_{\left(Q_{K_{1}} / V_{J_{1}, K_{1}}\right)}\right)^{\sim^{L_{J_{2}}} .}
$$

Here $K_{1}$ is as in 2.3 (note: ${ }^{w^{-1}} L_{K_{1}}=L_{K_{2}}$ ).

3. The results of this section are of independent interest, and are due to Curtis ([4]). They are needed to apply the results of $\$ 2$ to the duality operation.

3.1. Lemma. Let $w \in W,{ }^{w} L_{J_{2}}=L_{J_{1}},{ }^{w} \lambda_{2}=\lambda_{1}$, where $\lambda_{i} \in \operatorname{char}\left(L_{J_{i}}\right)$. Then $\lambda_{1}^{G}=\lambda_{2}^{G}$.

The idea of the proof is to show that the numbers $\left(\widetilde{\lambda}_{i}^{G}, \widetilde{\lambda}_{j}^{G}\right)_{G}$ are all the same for $i, j=1,2$. The proof in [3] (for the special case when $\lambda_{1}, \lambda_{2}$ are cuspidal) can be modified to work in the present situation.

The following is Lemma 2.5 of [4]. Then

3.2. LEMMA. Let $a_{J_{2}, J_{1}, K}=\left|\left\{w \in W_{J_{1}, J_{2}} \mid w_{K}=w_{J_{1}} \cap{ }^{w} W_{J_{2}}\right\}\right|$.

$$
\sum_{J_{2} \subseteq R}(-1)^{\left|J_{2}\right|} a_{J_{2}, J_{1}, K}=(-1)^{|K|}
$$


4. The first main result relates duality and the operations $\zeta \rightarrow \zeta_{\left(P_{J} / V_{J}\right)}$ and $\lambda \rightarrow \widetilde{\lambda}^{G}$. Part (1) is Theorem 1.3 of [4].

THEOREM. (1) $\left(\zeta^{*}\right)_{\left(P_{J} / V_{J}\right)}=\left(\zeta_{\left(P_{J} / V_{J}\right)}\right) *$ for $J \subseteq R, \zeta \in \operatorname{char}(G)$

(2) $\left(\tilde{\lambda}^{G}\right)^{*}=\left(\lambda^{*}\right)^{\sim G}$ for $J \subseteq R, \lambda \in \operatorname{char}\left(L_{J}\right)$.

We provide a sketch of the proof of (2). Let $J_{1}=J$. Using 2.4, 2.2, and then Lemma 3.1 (noting that $L_{K_{1}}={ }^{w} L_{K_{2}}$ by Proposition 2.6 of [3]) we have

$$
\left(\tilde{\lambda}^{G}\right)^{*}=\sum_{J_{2} \subseteq R}(-1)^{\left|J_{2}\right|} \sum_{w \in W_{J_{1}, J_{2}}} \lambda_{\left(Q_{K_{1}} / V_{J_{1}, K_{1}}\right)^{\sim^{G}}}
$$

The proof is then completed by applying Lemma 3.2 and 2.2 .

4.2 Theorem. The map $\zeta \rightarrow \zeta^{*}$, from $\operatorname{char}(G) \rightarrow \operatorname{char}(G)$ is an isometry of order two. In particular, $\zeta^{* *}=\zeta$ and $\pm \zeta^{*} \in \operatorname{Irr}(G)$, whenever $\xi \in \operatorname{Irr}(G)$.

In order to prove Theorem 4.2 , one first proves that $\left(\zeta_{1}, \zeta_{2}^{*}\right)_{G}=\left(\zeta_{1}^{*}, \zeta_{2}\right)_{G}$. It then suffices to prove $\zeta^{* *}=\zeta$. The key is to apply Theorem 4.1 part (1) to the expression for $\zeta^{* *}$. We have

$$
\begin{aligned}
\zeta^{* *} & =\sum_{J \subseteq R}(-1)^{|J|} \zeta_{\left(P_{J} / V_{J}\right)^{*} \sim G} \\
& =\sum_{J \subseteq R}(-1)^{|J|} \sum_{K \subseteq J}(-1)^{|K|} \zeta_{\left(P_{K}\right)^{G}}
\end{aligned}
$$

using 2.2. To finish the proof, note that $\Sigma(-1)^{|J|}$ summed over all $J$ such that $K \subseteq J \subseteq R$ is zero unless $K=R$.

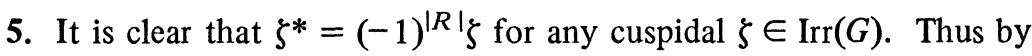
applying Theorem 4.1 part (2) we have:

5.1 Corollary. Let $\lambda \in \operatorname{Irr}\left(L_{\lambda}\right)$ be cuspidal. Then $\left(\tilde{\lambda}^{G}\right)^{*}=(-1)^{|J|} \tilde{\lambda}^{G}$.

Thus duality permutes (up to sign) the components of $\widetilde{\lambda}^{G}$. We can thus determine the "sign" of $\zeta^{*}$ as follows: $(-1)^{|J|} \zeta^{*}$ is in $\operatorname{Irr}(G)$ if $\zeta \in \operatorname{Irr}(G)$ is a component of $\tilde{\lambda}^{G}, \lambda \in \operatorname{Irr}\left(L_{J}\right)$ cuspidal. In particular, $\zeta \rightarrow \zeta^{*}$ permutes the principal series characters, i.e. the components of $\widetilde{\lambda}^{G}, \lambda \in \operatorname{Irr}\left(L_{\varnothing}\right)$. A more explicit result is known for the components $\zeta_{\varphi, q}$ of $1_{B(q)}^{G(q)}$ in a system of groups $\{G(q)\}$ of type $(W, R)$. Specifically, $\zeta_{\varphi, q}^{*}=\zeta_{\epsilon \varphi, q}$ where $\epsilon$ is the sign character of $W([4])$. 
Finally, consider the case $G=\mathbf{G}^{F}$ where $\mathbf{G}$ is a reductive algebraic group and $F: \mathbf{G} \rightarrow \mathbf{G}$ is a Frobenius map over $F_{q}$. Let $R_{\mathrm{T}}^{\mathrm{G}} \theta$ denote the DeligneLusztig generalized character of $G$ ( $\mathbf{T}$ an $F$-stable maximal torus of $\mathbf{G}, \theta$ a linear character of $\mathbf{T}^{F}$ ). It is natural to ask whether

$$
\left(R_{\mathrm{T}}^{\mathrm{G}} \theta\right)^{*}= \pm R_{\mathrm{T}}^{\mathrm{G}} \theta
$$

holds. The following suggests the answer is yes.

$$
\left(R_{\mathrm{T}}^{\mathrm{G}} \theta\right)^{*}(s)= \pm R_{\mathrm{T}}^{\mathrm{G}} \theta(s)
$$

for semisimple elements $s$ of $G$. The \pm sign in 5.3 does not depend on the particular element $s$ of $G$. The proof of 5.3 uses several results of [5]. (Note added in proof: The conjecture 5.2 has been proved by G. Lusztig.)

5.4 EXAMPLE. Let $G=\mathbf{G}^{F}$ as above, with (relative) Coxeter system $(W, R)$. Let $V$ be the set of unipotent elements of $G$ and let $\epsilon_{V}$ be the characteristic function of $V$. A recent result of Springer (Theorem 1 of [6]) ${ }^{1}$ shows

$$
\epsilon_{V}=q^{d} \sum_{J \subseteq R}(-1)^{|J|}\left|P_{J}\right|^{-1} 1_{V_{J}}^{G}
$$

where $d=\operatorname{dim}(\mathbf{G} / \mathbf{B})$, B a Borel subgroup of G. Applying Theorems 4.1 and 4.2 we have:

5.5 ThEOREM. (1) $\epsilon_{V}^{*}=\left(q^{d} /|G|\right) \rho_{G}$ where $\rho_{G}$ is the regular character of $G$.

(2) For $\xi \in \operatorname{Irr}(G)$,

$$
\frac{1}{\zeta(1)} \sum_{v \in V} \zeta(v)=q^{d}\left(\zeta^{*}(1) / \zeta(1)\right) .
$$

(3) For $\zeta \in \operatorname{Irr}(G),\left|\zeta^{*}(1)\right|_{p^{*}}=\zeta(1)_{p^{\prime}}$ where $p$ is the characteristic of $F_{q}$ and $n_{p^{\prime}}$ is the $p^{\prime}$ part of $n$.

(4) For $\zeta \in \operatorname{Irr}(G), 1 / \zeta(1) \Sigma_{v \in V} \zeta(v)$ is, up to sign, a power of $p$.

Part (4) of Theorem 5.5 confirms a special case of a conjecture of Macdonald (see [6]), namely the case when $q=p$ is prime.

\section{REFERENCES}

1. A. Borel and J. Tits, Groupes reductifs, Inst. Hautes Etudes Sci. Publ. Math. 27 (1965), 55-151.

2. C. W. Curtis, The Steinberg character of a finite group with a $(B, N)$-pair, J. Algebra 4 (1966), 433-441.

${ }^{1}$ The author is indebted to T. A. Springer for communicating both his results in [6] and the suggestion of G. Lusztig of combining them with duality. 
3. Reduction theorems for characters of finite groups of Lie type, J. Math. Soc. Japan 27 (1975), 666-688.

4. Truncation and duality in the character ring of a finite group of Lie type, J. Algebra (to appear).

5. P. Deligne and G. Lusztig, Representations of reductive groups over finite fields, Ann. of Math. 103 (1976), 103-161.

6. T. A. Springer, A formula for the characteristic function of the unipotent set of a finite Chevalley group (to appear).

DEPARTMENT OF MATHEMATICS, UNIVERSITY OF OREGON, EUGENE, OREGON 97403 